\title{
An Automatic Acquisition Method of Lead Planes Based on Particle Swarm Optimization
}

\author{
Yongmei Zhao, Jun Zhang, Mingfu Tuo, Li An, Hongmei Zhang \\ School of Science, Air Force Engineering University, Xi'an, Shaanxi 710051, China
}

\begin{abstract}
In a cooperative air combat, the whole command and control system (CCS) may lose control if the lead plane in the middle of the fleet is damaged or fails. In order to solve this problem, this article simulates a dynamic and global optimum CCS for the cooperative air combat with the particle swarm optimization (PSO) algorithm and automatically determines the lead plane by adding "lead bird" selection factors to the basic algorithm. Finally, the experiments verify this method is feasible and reliable.
\end{abstract}

Multi-plane air combat command and control structure is generally classified into three levels: organization, coordination and execution, which definitely specifies the authorities and ranks of command and control. When the lead plane in the middle of the fleet cannot command the fleet due to damages and malfunction, the whole command and control system (CCS) shall lose control ${ }^{[1]}$. Literature [2] suggests the airborne early warning (AEW) airplane to take the overall command and control centralized control in the whole theater of operations. Such command structure can solve the loss of control due to damages or failures of the lead plane, but it significantly reduce the autonomous offensive of single airplane because the whole data link requires more time to communicate when all commands are issued by the early warning airplane. In Literature [3], the author put forward the concept and structural frames of the cooperative air combat CCS. Literature [4] introduced a model of the air combat command and control system Eagle Vision used by US Army, but did not give the specific analysis thereof. The cooperative combat is very similar with the behaviors of bird groups ${ }^{[5]}$. Therefore, the article adopts swarm intelligence-based PSO algorithm to simulate an overall and dynamic cooperative air combat CSS and achieve automatic acquisition of lead plane by adding the "lead bird" selection factors to the basic algorithm.

\section{Automatic acquisition model of lead plane based on PSO}

\subsection{Air fleet system model based on PSO}

There is a mapping relation between PSO and fleet, so we can properly modify and expand PSO and determine the movement direction, speed and expected position of the individual using PSO of Reynolds model, in which the value of outside signals detected by the airplane is the adaptive value and the plane in the communication range as the perception neighborhood ${ }^{[6]}$. Meanwhile, calculate the movement position of the plane in real time with communication modes, communication cycles and sampling cycles, etc. The flight path should be planned if there is any barrier in the operation area and the size of the plane is considered. Individual optimization and overall optimization depend on the mission schedule. Accordingly, we can take PSO model as the behavior control model of the airplanes to coordinate and control movement behaviors of the airplanes, achieving swarm intelligence ${ }^{[7,8]}$.

The equation of PSO-based modeling is indicated as follows:

$$
\left\{\begin{array}{l}
V_{i}^{\text {exp }}(t+1)=w V_{i}(t)+c_{1} r_{1}\left(P_{b}-X_{i}(t)\right)+c_{2} r_{2}\left(P_{g}-X(t)\right) \\
V_{i}(t+1)=V_{i}(t)+\frac{1}{T}\left(V_{i}^{\text {exp }}(t+1)-V_{i}(t)\right) \\
X_{i}(t+1)=X_{i}(t)+V_{i}(t+1) \Delta t
\end{array}\right.
$$

Where $\operatorname{Vexp}^{\mathrm{i}}(\mathrm{t}+1)$ denotes the expected speed of the $\mathrm{i}^{\text {th }}$ plane at $\mathrm{t}+1$ time, $\mathrm{V}^{\mathrm{i}}(\mathrm{t})$ is the speed ${ }^{\mathrm{i}}$ of the $\mathrm{i}^{\text {th }}$ plane at $\mathrm{t}$ time ${ }^{\mathrm{ii}}, \mathrm{X}_{(\mathrm{t})}^{\mathrm{i}}$ denotes the position of the $\mathrm{i}^{\text {th }}$ plane at $\mathrm{t}$ time, $\mathrm{P}_{\mathrm{b}}$ denotes the optimal position of individual plane at a time, $\mathrm{P}_{\mathrm{g}}$ denotes the optimal position of swam planes at a time, $\mathrm{w}$ is an inertia factor of the algorithm, $c_{1}$ and $c_{2}$ respectively denote cognitive and social acceleration 
constants, $1 / \mathrm{T}$ is the expression of one-order inertia link of the plane and its controller, $\mathrm{r}_{1} \sim \mathrm{U}(0,1)$, $\mathrm{r}_{2} \sim \mathrm{U}(0,1)$ are two random variable independently from each other and $\Delta \mathrm{t}$ denotes the step size factor $^{[9]}$.

\subsection{Leadership acquisition model of lead plane}

\subsubsection{Concept of "lead bird"}

"Autonomous Boid" ${ }^{[0]}$ is improved on basis of Reynolds model by adding the concept of "lead bird". A true bird flock behavior model should include an external factor leadership. In the "lead bird" model, leader alteration is introduced, which is complementary with the alignment steering to keep the formation patter of the flockmates. However, the leadership depends on the position of Boid and irregularity of the swarm.

\subsubsection{Model for leadership acquisition of lead plane}

Firstly, it must estimate whether the plane is in the middle of the fleet or at the fleet edge. Assume $x_{i}$ is the position parameter of $i^{\text {th }}$ plane $b_{i}$, then

$$
x_{i}=\sum_{\forall b_{j} \in V_{i}} \frac{p_{j}}{m \times e}
$$

$x_{i} \in(0,1), V_{i}$ denotes the neighbouring set of $b_{i}$, $p_{j}$ denotes the position of $b_{j}, m$ denotes the number of neighbours of the neighboring set and e denotes the length of field of view for $b_{i}$. If $x_{i}$ is equal to $0, b_{i}$ is in the center of the flock; if $x_{i}$ is close to $1, b_{i}$ is close to the edge of the flock. If

$$
y_{i}=-\frac{\overrightarrow{k_{i}}}{\left|\overrightarrow{k_{i}}\right|} \times \frac{\overrightarrow{v_{i}}}{\left|\overrightarrow{v_{i}}\right|}
$$

$k_{i}$ denotes the cohesion direction of $b_{i}$ and Vi denotes the velocity direction of $b_{i}$. When $y_{i}$ is less than $0, b_{i}$ is at tail end of the flock; when $y_{i}$ is less than $0, b_{i}$ is at the front of the flock; the more $y_{i}$ is close to 0 , the nearer $b_{i}$ is in the center of the flock..

According to the Equations (2) and (3), it is not hard to get the determination function of the lead plane, namely the function of leadership alteration:

$$
z_{i}=\sum_{\forall b_{j} \in V_{i}} \frac{p_{j}}{m \times e}-\frac{\overrightarrow{k_{i}}}{\left|\overrightarrow{k_{i}}\right|} \times \frac{\overrightarrow{v_{i}}}{\left|\overrightarrow{v_{i}}\right|}
$$

The more the value of $z_{i}$ is close to 0 , the higher probability that $z_{i}$ becomes the lead plane.

\subsection{Establish target functions}

Take Ackley function as the target function to solve the value of fitness.

$$
\begin{gathered}
\min f(x)=-c_{1} \exp \left(-0.2 \sqrt{\frac{1}{n} \sum_{j=1}^{n} x_{j}^{2}}\right)-\exp \left(\frac{1}{n} \sum_{j=1}^{n} \cos \left(2 \pi x_{j}\right)\right)+c_{1}+e \\
x_{j} \in[-5,5], j=1,2, \cdots, n
\end{gathered}
$$

If $\mathrm{j}^{\text {th }}$ particle is determined as the lead plane, the particle has a certain decision-making authority and enjoys preferable right for update. Thus, take the updated values of the lead plane as modification factors in target function to $b$ modified subject to its limit value in each iteration and the target function is modified as follows:

$$
f(x)=0.5\left(-c_{1} \exp \left(-0.2 \sqrt{\frac{1}{n} \sum_{j=1}^{n} x_{j}^{2}}\right)-\exp \left(\frac{1}{n} \sum_{j=1}^{n} \cos \left(2 \pi x_{j}\right)\right)+c_{1}+e\right)+0.5 \text { fitnessgbest }(j)
$$

Where, fitnessgbest $(\mathrm{j})$ denotes the optimal value of a boid when the particle $\mathrm{j}$ is iterated. 


\section{Automatic acquisition algorithm of lead plane on basis of PSO}

\subsection{Algorithm flow chart}

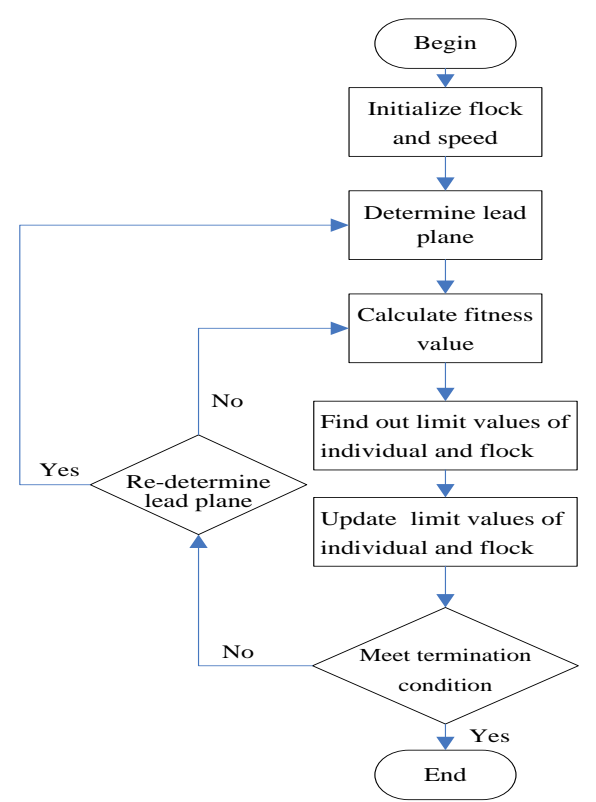

Fig.1 : Algorithm flow chart of automatic acquisition of lead plane

\subsection{Algorithm steps}

Step1: Initialization of parameters. Initialize $c_{1}$ and $c_{2}$ (two parameters in PSO), maxgen (iteration numbers) and sizepop(scale of particle swarms)

Step2:Generate initial particles and speed. Generate and initialize flocks at random: pop(i,:) $=5^{*}$ rands $(1,2)$; initialize the speed $V(i,:)=\operatorname{rands}(1,2)$.

Step3: Determine the lead plane. Traversing all particles, the plane with $\mathrm{z}_{\mathrm{i}}$ closest to 0 is determined as the lead plane in Equation (4).

Step4:Calculate the fitness of particles. fitness(i)=fun(pop(i,:)).

Step5: Calculate the initial value. The perfect fitness value of individual is zbest=pop(bestindex,:); the perfect fitness value as a whole is fitnesszbest=bestfitness.

Step6: Iterative optimization.Update the speed, $\mathrm{V}(\mathrm{j},:)=\mathrm{V}(\mathrm{j},:)+\mathrm{c} 1 * \operatorname{rand} *(\operatorname{gbest}(\mathrm{j},:)-\operatorname{pop}(\mathrm{j},:))+$ c2*rand*(zbest - pop(j,:)); V(j,find( $(\mathrm{j}(\mathrm{j},:)>\mathrm{Vmax}))=\mathrm{Vmax}$;

$\mathrm{V}(\mathrm{j}$,find $(\mathrm{V}(\mathrm{j},:)<\mathrm{Vmin}))=\mathrm{Vmin}$. Update the position, $\operatorname{pop}(\mathrm{j},:)=\operatorname{pop}(\mathrm{j},:)+0.5^{*} \mathrm{~V}(\mathrm{j},:)$; $\operatorname{pop}(\mathrm{j}$,find $(\operatorname{pop}(\mathrm{j},:)>\operatorname{popmax}))=\operatorname{popmax}$;

$\operatorname{pop}(\mathrm{j}$, find $(\operatorname{pop}(\mathrm{j},:)<\operatorname{popmin}))=$ popmin .

Step7: Update the optimal value of boid

if fitness $(\mathrm{j})<$ fitnessgbest $(\mathrm{j})$,gbest $(\mathrm{j},: \mathrm{)})=\operatorname{pop}(\mathrm{j},:)$; fitnessgbest $(\mathrm{j})=$ fitness $(\mathrm{j})$; Update the optimal value of flock, If fitness $(\mathrm{j})<$ fitnesszbest,

zbest $=\operatorname{pop}(\mathrm{j},:)$,fitnesszbest $=$ fitness $(\mathrm{j})$.

Step8: Determine whether iteration numbers have been reached, if not, go to Step 9; otherwise terminate the algorithm.

Step9: Determine whether the lead plane is needed to be determined once again, if yes, go to Step 3; otherwise, go to Step 4.

\section{Simulation experiment}

It is assumed that the two parameters of PSO are c1 $=1.49445$; $c 2=1.49445$; iteration numbers maxgen=200; size of PSO sizepop=20; and in the target function, $c 1=20, e=2.71282$. When the simulation experiment is carried out in matlab, firstly carry out simulation experiment on the fleet integrated with the automatic acquisition model of the lead plane (and during cooperative air combat when the lead plane is damaged), the results are indicated in Fig.2: 

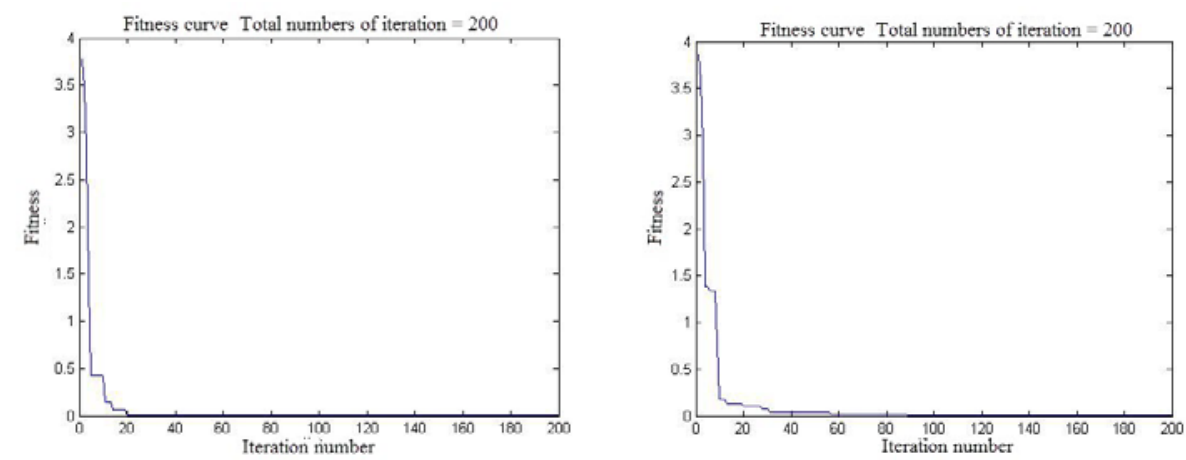

Fig.2: Simulation as the lead plane is damaged

Fig.3: Simulation with automatic acquisition model of the lead plane

Simulation results: zbest $=0.01010 .0351$, a steady result has been obtained from the 22th iteration. Thus we can see that this algorithm has a remarkable optimization result, but it is easily involved into the solution of local optimized values. Then carry out simulation test on the fleet with automatic acquisition model of the lead plane and the result is shown in Fig.3. The simulation results: zbest $=-0.00350 .0002$, a steady result has been obtained from the 86th iteration and No.34 plane is determined as the lead one. We can see from the above results that the model with containment of leadership can get a better optimization and convergence effect and also reduce the possibility of involvement in local optimal solution.

\section{Conclusion}

This article uses traditional PSO to simulate cooperative air combat of the fleet. The cooperative air combat under ideal conditions meet the principle of avoidance of repetition, overall optimization and balanced distribution while taking consideration of the information share with other friendly planes and tactical and fire coordination. However, some containment of leadership, namely guidance of the lead plane is needed in actual combat. For this reason, the automatic acquisition model of the lead plane is added on basis of traditional PSO. It is verified by simulation tests that this method is feasible and reliable to a certain degree, and thus significant to improve the flexibility and timeliness of the command and control system.

\section{References}

[1]Zhaowang Fu, Yingxin Kou, Lei Yu and Zhanwu Li. Demand analysis on cooperative air combat mode and capabilities of manned/unmanned fighters [J]. Fire Control \& Command Control, 2012,12(1):74-77.

[2]Jinxing Liu and Ming'an Tong. Some technical issues arising from control and command of cooperative air combat for multi-intelligent combat planes [J]. Electronics Optics \& Control, 2007,14(1):155-157.

[3]Jinxing Liu and Ming'an Tong. Command and control system for air-to-air cooperative combat [J]. Fire Control \& Command Control, 2001, 26 (4):43-46.

[4] Levis A H. Time sensitive control of air combat operations [D].Cebter of Excellence in Command, Control, Communication and Intelligence. George Mason University,1998.

[5] Chushi Feng. Swarm intelligence optimization algorithm and its application [D]. University of Science and Technology of China, 2009.

[6]Jianchao Zeng and Songdong Xue. Modeling and simulation of swarm robot system [J]. Journal of Computer Simulation, 2010,22(6):1327-1330.

[7]Molly M K.Performance analysis using stochastic Petri nets[J]. IEEE Trans on Computer,1982,SE-11(9): 913-917. 
[8] Zhili Tang and An Zhang. Studies on estimation theories and methods for threats in battlefield[J]. Fire Control \& Command Control, 2011, 36(9):2-4.

[9] Jin Li, Songdong Xue, Jianchao Zeng and Yinzhang Guo. Studies on communication modes in target search of swarm robots [J]. Journal of Taiyuan University of Science and Technology, 2011, 32(4):265-268.

[10] Yao Guo. Studies on simulation of attack avoidance behaviors on basis of biological characteristics of bird flock [D]. Computer Science \& Technology, Central South University, 2011. 